\title{
Improving quality of life for people with dementia in care homes: making psychosocial interventions work ${ }^{\dagger}$
}

\author{
Vanessa Lawrence, Jane Fossey, Clive Ballard, Esme Moniz-Cook and Joanna Murray
}

\section{Background}

Psychosocial interventions can improve behaviour and mood in people with dementia, but it is unclear how to maximise their effectiveness or acceptability in residential settings.

\section{Aims}

To understand what underlies the successful implementation of psychosocial interventions in care homes.

\section{Method}

Systematic review and meta-synthesis of qualitative research.

\section{Results}

The synthesis of 39 qualitative papers revealed that beneficial psychosocial interventions met the needs of people with dementia to connect with others, make a meaningful contribution and reminisce. Successful implementation rested on the active engagement of staff and family and the continuing provision of tailored interventions and support. This necessitated staff time, and raised issues around priorities and risk, but ultimately helped redefine staff attitudes towards residents and the caregiving role.

\section{Conclusions}

The findings from the meta-synthesis can help to inform the development and evaluation of psychosocial interventions in care homes and support their widespread implementation in clinical settings.

\section{Declaration of interest}

None.
Twenty-five million people worldwide have dementia, including 700000 in the UK, of whom an estimated 250000 live in care homes. ${ }^{1}$ Dual concerns about the prevalence of psychiatric and behavioural symptoms, present in $80 \%$ of people with dementia in care homes, and the overprescription of antipsychotic medication have led to a renewed emphasis on non-pharmacological interventions. ${ }^{2}$ Non-pharmacological interventions use a wide range of approaches, characterised as behaviour-, emotion-, stimulation- or cognition-oriented, ${ }^{3}$ that aim to improve quality of life and maximise function in the context of existing deficit. Training and support interventions for nursing home staff have proven to be an effective alternative to antipsychotic medication in managing disruptive behaviour in people with dementia. ${ }^{4} \mathrm{~A}$ recent appraisal of the quantitative evidence in this area concluded that behavioural management techniques, cognitive stimulation and exercise can improve key mental health outcomes such as agitation and depression. ${ }^{5}$ However, none of these interventions has achieved widespread implementation in care home settings and there is no coherent picture of how to maximise their effectiveness or acceptability. Other 'promising' interventions have been found to have a positive yet not always statistically significant impact in controlled trials, ${ }^{5}$ with participants showing marked variation in responses. ${ }^{6}$

Evaluating complex interventions poses unique challenges and revised Medical Research Council guidelines highlight the necessity not only of measuring outcomes but also of exploring the ways in which the intervention under study is implemented. Qualitative methods can be used to elucidate this process and to identify the effective components of an intervention and the context in which it can be optimised. ${ }^{7}$ Individual qualitative studies provide insight into how different psychosocial interventions are used and experienced within residential settings. Integrating these findings in a qualitative meta-synthesis promises to enhance their impact on health policy and clinical practice ${ }^{8,9}$

†See editorial, pp. 342-343, this issue. and help us to understand and overcome the potential obstacles to implementing psychosocial interventions as part of routine care. To our knowledge this is the first systematic review and meta-synthesis of the qualitative evidence in this area.

\section{Method}

Meta-synthesis is a method of integrating findings from qualitative studies. The aim is not to reduce data to summary form, but to amplify it through interpretation. ${ }^{10}$ Thus, the purpose is to achieve a level of understanding and conceptual development that is greater than that attained from the individual empirical studies alone. ${ }^{9}$ Multiple methods for synthesising qualitative research exist and it is argued that the purpose of the meta-synthesis and the nature of the findings should direct the approach used. ${ }^{10}$ For instance, we aimed to go beyond the description and summary associated with conventional narrative reviews of qualitative research, but were mindful that the wide-ranging level of interpretation of data within the individual studies might preclude a meta-ethnography approach. Meta-ethnography is a widely used method of synthesising qualitative data, but requires all findings to be in the form of concepts or metaphors. ${ }^{11}$ We considered the range in level of interpretation and methodological design typical of qualitative health research and did not wish to restrict the synthesis on this basis. Thus, we used analytical devices such as constant comparison and the creation of taxonomies to develop a conceptual synthesis of the successful implementation of psychosocial interventions in care homes. ${ }^{10}$

\section{Identification and selection of papers}

Systematic review methodology was used to identify relevant articles. ${ }^{12}$ A search strategy combining medical subject headings and text words relating to dementia, care homes, psychosocial interventions and qualitative research was devised and adapted for seven electronic databases. We carried out searches in Medline (1995 to January 2011), PsycINFO (1995 to January 2011), British Nursing Index (BNI) (1995 to January 2011), EMBASE (1995 to 
January 2011), CINAHL (1995 to January 2011), Applied Social Sciences Index and Abstracts (ASSIA) (1995 to January 2011), Allied and Complementary Medicine Database (AMED) (1995 to January 2011) and System for Information on Grey Literature in Europe (SIGLE) (1995 to March 2005). The study was restricted to English-language articles that reported qualitative research regarding the use and effectiveness of psychosocial interventions for people with dementia in care homes, from the perspective of people with dementia, relatives or care staff. Participants with any type of dementia residing in any type of residential facility were considered.

The psychosocial interventions comprised therapies that were derived from psychological or psychosocial models designed to improve outcomes in people with dementia and the search terms were discussed and agreed upon by the research team. They included: person-centred care, person care planning, emotion oriented, validation, communication, interaction, resolution, engagement, cognitive stimulation therapy, cognitive training, reality orientation, reminiscence, life stories, life review, life history, activity, exercise, psychosocial, psychotherapy, psychoanalysis, counselling, behaviour therapy, creative therapies, art, mural, animal-assisted therapy, pets, animals, leadership, education, dolls, music therapy, drama therapy, dance therapy, Jabadoo, and self-maintenance therapy. Qualitative research is poorly indexed in databases, ${ }^{13}$ so a combination of thesaurus terms and free-text terms was used to maximise the recall of potentially relevant studies. ${ }^{14}$ We augmented the electronic search by scanning reference lists and hand-searching relevant journals and publications of influential research groups in the field (such as Journal of Dementia Care, Alzheimer's Society, Social Care Institute for Excellence, Stirling University Dementia Services Development Centre).

Studies in which interventions were delivered to care staff were included (e.g. training programmes) if a primary aim was to improve outcomes for the person with dementia. The review also included papers that focused on the implementation of psychosocial interventions. However, purely descriptive or theoretical studies that did not present empirical data on the use of psychosocial interventions in care homes were excluded. In total, 2894 papers were identified; after adjusting for duplicates the abstracts of 2384 papers were screened and 2261 were excluded on the basis that they did not meet the study criteria. The full texts of the remaining 123 articles were then independently assessed for eligibility by two reviewers (V.L. and J.M.). Agreement was measured using Cohen's Kappa $(\kappa)$ and disagreements were resolved by discussion through referring to the eligibility criteria and the original studies where necessary.

\section{Quality appraisal}

Two experienced qualitative researchers (V.L. and J.M.) independently assessed relevant papers for methodological quality using the Critical Appraisal Skills Programme (CASP) checklist. ${ }^{15}$ The broad quality criteria within this checklist provided a flexible method of assessing the rigour, credibility and relevance of the heterogeneous set of studies. The reviewers compared their appraisal assessments and reconciled any differences in judgements through debating the rationale for their decisions. Studies that did not use qualitative methods of data collection and analysis or did not give an account of these procedures were excluded at a preliminary screening stage. In qualitative synthesis there is a tension between inclusiveness and quality. ${ }^{16}$ We opted for an inclusive strategy and, in common with other synthesis ${ }^{9}$ the quality appraisal process was not used as a criterion to exclude studies but allowed us to judge the value of papers with respect to their contribution to the synthesis. Themes were only included in the meta-synthesis if they were supported by data from at least one article judged to be of reasonable quality. Scores on the CASP checklist range from zero to ten and a score of seven or higher was defined as the threshold for 'reasonable quality' at the outset of the study.

\section{Data extraction and synthesis}

Data concerning the type of intervention, aims, participant characteristics, setting and methods of evaluation used in each study were recorded as essential contextual information for the synthesis. Subsequently, a description of the main concepts derived from each paper was entered into a table using the original authors' own words or a paraphrase. ${ }^{17}$ Efforts were made to remain faithful to the meanings and concepts within each study. Authors' interpretations, explanations and recommendations were also extracted from the paper where relevant to the research question. By examining the concepts and interpretations within the table it was possible to identify shared constructs across studies and to note areas of discordance. The themes were combined using an interpretive method of meta-data synthesis, grouping themes where they had greatest explanatory power. This enabled the construction of a taxonomy that categorised findings in three domains: beneficial elements of a psychosocial intervention; conditions required for successful implementation; and challenges to successful implementation (see Appendix). The taxonomy was developed inductively from the findings and the properties and dimensions of each domain were explicated through moving back and forth between findings and taxonomy (a process similar to the constant comparison method used in primary qualitative research)..$^{10}$ All members of the research team commented on the emerging typology and interpretations were discussed between V.L., J.F. and J.M at regular intervals. All authors have substantial experience of working with people with dementia and their varied disciplinary and methodological expertise helped to produce insights and generate different questions to be asked of the primary research. Finally, the synthesis was written and revised several times until a coherent whole was formed.

\section{Results}

A total of 39 papers, pertaining to 35 individual studies, were included in the synthesis $(\kappa=0.68) .{ }^{18-56}$ The study selection process and reasons for exclusion are shown in Fig. 1. Using the CASP checklist, 29 of the 39 papers were rated as of least reasonable quality $(\mathrm{CASP} \geqslant 7)$. The level of agreement between reviewers was good (Weighted $\kappa=0.66$ ). Common weaknesses of the ten remaining papers $(\mathrm{CASP}<7)^{21,29,30,33,36,37,39,41,46,47}$ included a failure to demonstrate that the data analysis was sufficiently rigorous or to critically examine the researcher's role or potential bias. The stated methodology and methods of data collection and analysis of the included studies are specified alongside other study characteristics in online Table DS1. The 35 studies were conducted in the following countries: 13 in the USA; 7 in Sweden; 5 in England; 5 in Canada; 2 in Australia; 1 in Ireland; 1 in Japan; and 1 in Norway. Thus, the research was undertaken in a range of care settings, including special care units, nursing homes and assisted living facilities, suggesting variations in philosophies, staffing patterns and structures. Enormous diversity exists in the nature of treatments included under the rubric 'psychosocial' and this was evident in the 35 studies reviewed. Study interventions were broadly categorised as: music interventions $(n=5$ studies) ${ }^{20,29,42-45,49}$ exercise and other therapeutic activities $(n=4) ;{ }^{23-25,30,38,51}$ reminiscence $(n=4) ; ;^{26,34,41,48}$ models of dementia care $(n=4) ; 27,33,46,56$ communication strategies $(n=3) ;^{19,21,54}$ staff 
training and supervision $(n=3) ;^{31,40,53}$ methods of orientation $(n=2) ; 22,37$ animal interventions $(n=2) ;{ }^{35,36}$ nature related activities $(n=2)^{39,47}$ and other $(n=6) .^{18,28,32,50,52,55}$

The research studies reported a wide range of positive outcomes for people with dementia including improved mood, communication, engagement, occupation, behaviour and quality of life. Researchers examined these outcomes, and drew conclusions about the beneficial elements of psychosocial interventions and the characteristics that might facilitate or obstruct their implementation, through exploring the perceptions and experiences of staff, relatives and people with dementia. Multiple methods of data collection were used, including interviews, focus groups, participant/non-participant observation and staff records, and many of the research studies triangulated multiple data sources to enhance the credibility of the findings. ${ }^{57}$

\section{Beneficial elements of a psychosocial intervention}

Beneficial elements of the interventions were identified, first, with respect to people with dementia and, second, with respect to care staff (Appendix). These will be discussed in turn.

\section{Focus on people with dementia}

One of the principal features of interventions that were perceived to be beneficial was that they enabled people with dementia to 'connect with others'. In the first instance, they created a tangible line of communication. Music, animals, dolls and 'remembering boxes' all provided a focal point that stimulated conversation between people with dementia and staff, relatives and other residents. ${ }^{20,29,35-37,41,49,52}$ Interventions involving volunteers from outside the home proved especially valuable in allowing people with dementia to form relationships beyond the normal institutional surroundings. ${ }^{35,36,50}$ Therapeutic interventions involving music and dance afforded the additional benefit of enabling individuals to express emotions and show feelings towards one another. ${ }^{20,24,25}$ Greater mutuality was seen as music and singing enhanced communication and cooperation. ${ }^{44}$ Gottell et al reported that singing during caregiving activities created an implicit understanding between people with dementia and staff that reduced the need for verbal instructions. ${ }^{44}$ Finally, taking part in activities with other residents, or in some cases simply being present without participating, provided individuals with a valued sense of inclusion. ${ }^{38,52,56}$

A second distinctive feature of interventions perceived to be beneficial was that they enabled people to feel that they were making a 'meaningful contribution'. Individuals demonstrated pride in passing on values and experiences during structured reminiscence about their lives. ${ }^{26,48,50}$ An enhanced ability to contribute to and initiate conversations engendered a sense of control, ${ }^{41}$ and taking responsibility for a doll or animal brought a sense of purpose to residents' daily lives. ${ }^{35,52}$ Other meaningful activities included those that reflected prior roles or pastimes such as attending church. ${ }^{20,56} \mathrm{~A}$ third, allied characteristic of beneficial interventions was that they provided an 'opportunity to reminisce. This could be spontaneous, with evidence of music, dancing, animals and dolls prompting residents to recall memories, ${ }^{29,35,36,45,52}$ social skills and procedures, ${ }^{25}$ or planned, with structured reminiscence programmes facilitating focused and insightful thinking. ${ }^{48}$ Moreover, the creation of 'memory notebooks' and 'remembering boxes' provided concrete memory aides that assisted people with dementia in sharing memories and life stories with those around them.

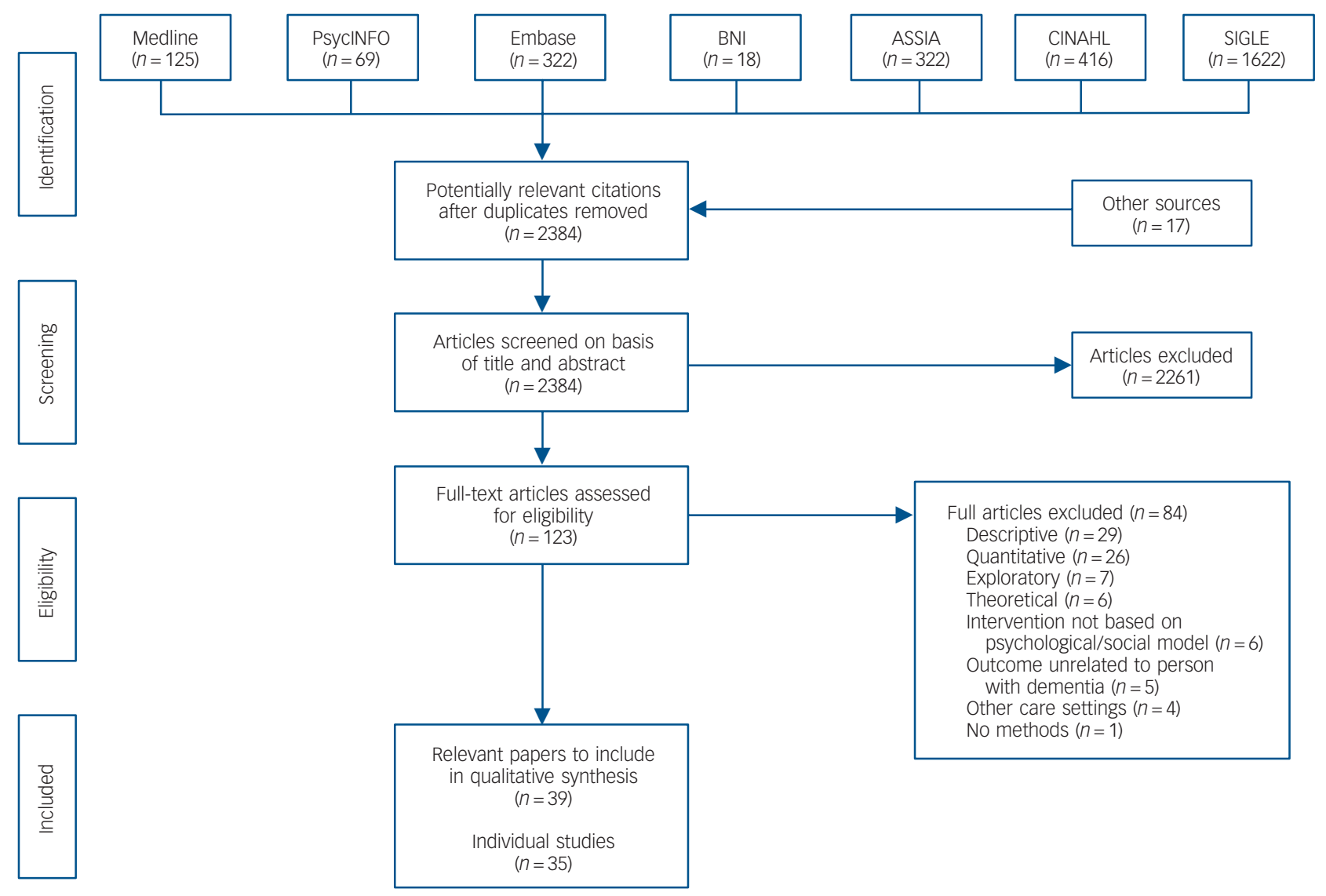

Fig. 1 Study selection process. 


\section{Focus on staff}

Staff across the studies agreed that from their own perspective psychosocial interventions were invaluable in helping them to 'see beyond the illness'. Often, this was manifested in the view that getting to know the person assisted staff in understanding and responding to behaviours that they had previously found challenging. ${ }^{26,32}$ This was achieved through life-review work $^{26,41}$ or the use of formal assessment measures ${ }^{40}$ that enabled staff to learn the personal histories of residents. Seeking information from family members not only proved expedient in getting to know personal characteristics, accomplishments, values and preferences, but also enhanced the care staff's appreciation of the resident as a person embedded in a family context. ${ }^{28,31}$ Ultimately, knowledge of the individual and their family helped staff to relate on a more personal level and develop a more meaningful relationship with the person with dementia.

A variety of other methods were used to reduce the distance between professional caregivers and patients. Dramatised vignettes and role-plays were a powerful catalyst in allowing staff to imagine what life must be like for people with dementia in care homes. ${ }^{31}$ Palo-Bengtsson \& Ekman observed that simply sharing experiences during dance events generated a new psychological closeness between residents and staff. $^{23}$ Special care units demonstrated expertise in dismantling 'us' and 'them' relationships; efforts were made to eliminate separate areas for staff and people with dementia and residents were encouraged to support each other and contribute to aspects of daily living rather than just being cared for by staff. ${ }^{27,56}$ Thus, interventions perceived to be beneficial helped staff to see the person beyond the dementia, but also assisted practitioners in broadening their concept of the caregiving role. Moreover, special care units where staff members played multiple and integrated roles, for example sharing responsibility for resident care, housekeeping and formal activities, encouraged staff to respond to the social and emotional, as well as the physical and medical needs of residents. ${ }^{27,46}$

In attributing a greater range of meaning to residents' behaviour, practitioners began to consider the importance of their own professional approach. Beneficial interventions fostered critical reflection in staff regarding how their own approach to care interactions might influence resident's behaviour and reactions. ${ }^{31,40,41}$ As a result the focus shifted from the emphasis staff placed on the time pressures in the workplace to the importance of making time to help the person with dementia feel comfortable. ${ }^{31,40}$ Supervision provided a valuable forum for staff to reflect on their caregiving style. ${ }^{40}$ This appeared most beneficial when discussion extended to practical problems, rather than considering theoretical principles alone, and when staff were able to talk through the issues that arose from applying the intervention in their everyday work. ${ }^{26,40,53}$ Research methods that encouraged staff to share their perceptions of the interventions with each other provided another useful opportunity to discuss barriers to implementation. ${ }^{33,55}$

\section{Conditions required for successful implementation}

The research studies demonstrated that people with dementia were entirely reliant on staff to access certain interventions, such as therapeutic gardens and listening to music, and suggested a shared expectation that staff would decide what to do, when to do it and what was allowed (Appendix). ${ }^{20,38,39,47}$ Certainly, carehome staff played a crucial role in initiating, directing and maintaining activities. Structure provided people with dementia with a sense of security and direction, although Kovach \& Henschel observed that therapeutic activity groups with less structure and smaller numbers allowed greater opportunities for self-expression and a focus on individual needs. ${ }^{30}$ Knowing the person emerged as one of the key benefits and determinants of a successful intervention. Staff not only used their knowledge of the individual to provide appropriate encouragement and motivation, ${ }^{30,34,38,47,51}$ but also to reassure residents and help them to feel comfortable and secure during interventions involving dance, music and reminiscence. ${ }^{20,24,25,45,48}$ Knowing the person also proved invaluable in offering music and activities that were tailored to the preferences of the individual ${ }^{29,38,42,49}$ and the residents' physical and cognitive abilities. ${ }^{23,25,37,40,48,54}$ Finding the right level of complexity was considered important, but sometimes difficult to achieve. In a structured reminiscence programme involving nine people with moderate dementia, tangible cues to focus memory replaced psychomotor tasks, which were found to be too difficult. ${ }^{48}$ Conversely, the simple design of a 'memory notebook' and the reliance on patients' distant rather than recent memory for dancing were seen to contribute to their successful implementation. ${ }^{37}$

Interacting with family members was found to assist staff in getting to know the likes, dislikes and capabilities of the person with dementia ${ }^{20,49,55}$ and promoted mutual appreciation and respect. ${ }^{34,40,55}$ Several studies reported creative methods of stimulating communication between relatives and staff such as 'action groups ${ }^{55}$ and 'family biography workshops. ${ }^{34}$ Long-Foley et al concluded that the active involvement of family members in decision-making, monitoring of case management and supporting staff ideas was critical to the successful management of behavioural problems in special care units and should be fostered by encouraging participation in care planning (by conference call and evening meetings if necessary), activity planning, support groups and assisting staff with caregiving (if desired) ${ }^{28} \mathrm{~A}$ further condition for successful implementation was scope for the intervention to be delivered flexibly to accommodate the range of abilities and behaviour. This included flexibility regarding the pace of the intervention $^{38,54}$ and the required level of engagement, for example supporting residents in listening to the music if they did not wish to dance. ${ }^{24}$ Finally, there was also a suggestion that activities should occur across a sufficiently prolonged period to allow residents to develop confidence in the intervention, for example enabling people with dementia to become familiar with the dog and volunteer in animal-assisted therapy. ${ }^{27,35}$

\section{Challenges to successful implementation}

Many of the interventions demanded extra work, reallocation of staff time and flexibility (Appendix). For instance, the numerous practical prerequisites to dance events ${ }^{24}$ and attending 'action group' meetings ${ }^{55}$ or implementing music interventions ${ }^{49}$ all took time. Mostly, staff accepted this as a requisite feature of the intervention that was justified by the outcomes it produced, ${ }^{24,33,49,53,55}$ but there were instances where low staffing levels restricted the use of psychosocial interventions, ${ }^{49,55}$ denied staff the opportunity to experiment with or adjust to a new way of working ${ }^{33,53}$ or prompted the alternate use of pharmacological interventions. ${ }^{32}$ In a minority of cases, discussed below, staff did not feel that the benefits of the intervention warranted the extra workload. ${ }^{39,47}$ In response to concerns about staff pressures, several studies stressed that activities such as singing, music and spending time outdoors had the potential to be incorporated into normal practice and the everyday care of people with dementia without placing extra demands on staff time..$^{20,39,42}$

There was a general sense that care professionals were being asked to implement psychosocial interventions against a 'changing landscape' of lower staff to resident ratios and heavier, more complex care needs among residents. ${ }^{32,46}$ This seemed to reinforce 
a focus among care staff on 'priority needs' whereby managing behaviour took precedence. There was a strong emphasis on preventing or avoiding behavioural outbursts through accomplishing tasks as quickly as possible, for example a focus on getting residents showered, rather than on how staff could best help residents during showering. ${ }^{19,28,32,51}$ Priority was given to aspects of physical care and safety rather than promoting autonomy, and behaviours perceived as being potentially harmful were actively discouraged. ${ }^{47}$ Risk of physical harm, falling or wandering were all considered barriers to implementing activities and exercise. ${ }^{27,32,51}$ Attitudes of individual members of the care team presented further challenges to implementing psychosocial interventions. Some staff members were uncomfortable participating in the intervention itself, stating that they disliked singing, dancing or going out into the garden. ${ }^{24,39,42}$ Kolanowski et al reported scepticism and a general lack of knowledge about the effectiveness of non-pharmacological interventions among care professionals who were not currently involved in their use. ${ }^{32}$ However, staff in only 2 of the 35 studies in this review questioned the therapeutic value of the intervention under study, commenting, for example, that nature-related activities appeared quickly forgotten. ${ }^{47,55}$ Finally, there was evidence among some staff of a resistance to interventions of any sort, with staff finding meetings with researchers from 'The University' threatening ${ }^{55}$ and believing that they had 'nothing more to learn. ${ }^{53}$

\section{Discussion}

\section{Main findings}

The meta-synthesis identified a number of key issues that, if addressed, would help to enable the widespread implementation of psychosocial interventions in care homes. This includes the importance of providing individualised interventions that are tailored to the person's preferences and abilities and of obtaining the full support of care staff. Staff members enable access to psychosocial interventions and provide essential encouragement and reassurance. Yet it is apparent that pressures on staff time, an associated focus on 'priority needs' and a preoccupation with risk present enduring barriers to implementation. The findings offer unanimous support for the principles of person-centred care ${ }^{58}$ and have important implications for clinical practice and research, which will now be discussed.

\section{Key issues in the successful implementation of psychosocial interactions}

Although it is self-evident that psychosocial interventions require the involvement of care staff, the synthesis underlines the absolute necessity of their commitment, knowledge and skills. Too often staff members are reluctant participants in dementia care training. ${ }^{59}$ The meta-synthesis suggests an initial scepticism about non-pharmacological approaches ${ }^{32}$ that needs to be addressed prior to implementation. It is essential to demonstrate that psychosocial interventions are a valuable option. Collaborative approaches that acknowledge staff perspectives and expertise from the outset offer an important method of engagement. ${ }^{60}$ This involves listening to any preferences or concerns that staff members have about the intervention in question. Finally, it is essential to obtain the full support of managers given their role in facilitating interventions and effecting cultural change within the home.

The synthesis attributes equal importance to the collaboration of relatives who help staff to get to know the person with dementia. Workshops and groups proved an effective method of fostering their involvement and ultimately enhanced cooperation and trust between relatives and the care team. This joint input, alongside flexibility in timing, duration and intensity, helped implement psychosocial interventions that were relevant to the interests, abilities and willingness of the individual. Of course the challenges involved in delivering a flexible, personalised intervention are magnified in controlled trials and this does not always occur, which may explain some of the ambiguous results reported. ${ }^{61}$ A notable exception is the work of Cohen-Mansfield and colleagues ${ }^{62}$ that demonstrated the efficacy of systematic individualised non-pharmacological interventions for decreasing agitation, and increasing pleasure and interest, in nursing home residents with dementia. We concur that tailoring psychosocial interventions to individual profiles enhances their clinical usefulness.

However, the meta-synthesis indicates that the successful implementation of psychosocial interventions is also a question of resources and time. Organisational support is necessary to enable care staff to sustain good practice, for example modifying work schedules, providing practice opportunities and changing policy or treatment guidelines. ${ }^{63}$ Training and mentoring programmes can require a substantial time commitment. Again, it is important to justify this investment through educating managers and care staff on the benefits of psychosocial interventions, not only for improving residents' behaviour and mood, but also for creating more efficient care practices within the home. Yet the current climate of financial constraints demands that attention is also given to how psychosocial interventions, be it therapeutic activities, reminiscence work or staff training, can be embedded into daily care. Examples within the synthesis include playing individual's favourite music during morning care or enjoying meals outdoors. To a large degree, this requires educating staff on person-centred practices that recognise, respect and provide opportunity for individual's self-expression. Equally, it is important that staff take a person-centred approach to assessing risk that does not simply identify every person with dementia as being at equal risk. ${ }^{64}$ The synthesis suggests that concerns around risk prevent staff from implementing certain activities. It is vital to challenge these perceptions and to encourage staff to consider, alongside any physical risks, the negative psychological effects of not being able to participate in meaningful activities. ${ }^{64}$

The findings are unequivocal that psychosocial interventions must be anchored in person-centred care. The synthesis underscores the fundamental importance of connecting with others and the benefits derived from social contact of different forms. Moreover, the desire to make a meaningful contribution and to reminisce mirror Kitwood's psychological needs of occupation and identity. ${ }^{58}$ Although no conclusions can be drawn about the relative effectiveness of the different interventions, the synthesis suggests that some psychosocial interventions are more likely to achieve these aims than others. For instance, reminiscence sessions provided opportunities to interact, to pass on values and experiences and to recall memories. This afforded multiple benefits; the challenge for controlled trials is how to measure the respective effects of the different active ingredients. Additionally, the synthesis offers support for certain procedures such as the use of volunteers, to allow residents to have social contact beyond the home, and concrete memory aids, which supported people in sharing memories with others. With respect to formal training for care staff, role-plays, videos and vignettes emerged as an effective teaching method, as illustrated elsewhere. ${ }^{59}$ A striking finding of the synthesis was that merely participating in psychosocial interventions, such as life-review work or music events, helped staff to see beyond the symptoms of dementia and to broaden their conceptualisation of the caregiving role. Yet, the findings also reinforce the importance 
of providing mechanisms for staff to reflect on their practice. ${ }^{59}$ Future intervention studies need to consider how supervision, peer support or audit practices can support staff without placing excessive burdens on resources.

\section{Further research}

Overall, the meta-synthesis presented a positive picture in which people with dementia, family and staff members derived important benefits from psychosocial interventions. However, further well-designed research is needed to strengthen the evidence base for psychosocial interventions in long-term care. ${ }^{5}$ Randomised controlled trials of complex interventions could be enhanced by integrating outcome evaluations with process evaluations that utilise qualitative methods. ${ }^{7}$ For example, future trials could include focus group discussions with care staff to understand and inform the process of implementation and to increase their collaboration in the research programme. The revised implementation plan for the National Dementia Strategy ${ }^{65}$ and parallel review of antipsychotic medication for people with dementia ${ }^{66}$ afford a unique opportunity to move forward on a national basis and develop a better trained workforce that is skilled in delivering psychosocial interventions and care. Combining quantitative and qualitative methods in clinical trials to identify 'what works' and 'how and why it works' in different circumstances can help to develop and deliver effective psychosocial interventions in real clinical settings.

\section{Strengths and limitations}

The study used rigorous methods for systematic review that included a comprehensive search of seven electronic databases, relevant journals and publications of influential research groups using predetermined criteria. Qualitative meta-synthesis offers a systematic, relevant overview of international qualitative research while retaining much of the detail that individual studies provide. However, there is a risk that synthesising across qualitative studies could compromise the integrity of the individual projects, and their emphasis on context and holism. ${ }^{8}$ We are mindful that this review identified a heterogeneous set of studies ranging in care setting, intervention type and methodological design. For this reason we provide comprehensive details about the primary studies in Table DS1 to convey the context of the study. Findings were also compared with reference to the study context and efforts were made to identify conflicting results. We also recognise that the sample size is large for a meta-synthesis, but are satisfied that the scope of the review was sufficiently focused and believe that the range of data assisted in identifying the properties and dimensions of the key concepts. As a consequence, the analytical themes presented in the synthesis offer a high level of conceptual thinking about the implementation of psychosocial interventions that can be applied across groups and settings.

\footnotetext{
Vanessa Lawrence, PhD, Institute of Psychiatry, King's College London; Jane Fossey, MSC, CPsychol, Oxford Health NHS Foundation Trust, Oxford; Clive Ballard Fossey, MSC, CPsychol, Oxford Health NHS Foundation Trust, Oxford; Clive Ballard,
MD, Wolfson Centre for Age-Related Diseases Clinical Trials Team, King's College $\mathrm{MD}$, Wolfson Centre for Age-Related Diseases Clinical Trials Team, King's College
London; Esme Moniz-Cook, PhD, Institute of Rehabilitation, University of Hull, and Humber NHS Foundation Trust, Hull; Joanna Murray, BA, Institute of Psychiatry, King's College London, UK

Correspondence: Clive Ballard, Wolfson Centre for Age-Related Diseases Clinical Trials Team, Guy's Campus, King's College London, London SE1 1UL, UK. Email: clive.ballard@kcl.ac.uk

First received 26 Aug 2011, final revision 24 May 2012, accepted 14 June 2012
}

\section{Funding}

This review was funded as part of a 5-year National Institute for Health Research (NIHR) programme grant.

\section{Appendix}

\section{Taxonomy of findings pertaining to implementation} of psychosocial interventions in care homes

Beneficial elements of a psychosocial intervention

(a) Focus on people with dementia

(i) Connecting with others:

line of communication $20,29,35-37,41,49,52$

bringing the world in $35,36,50$

mutual understanding $20,23,25,44$

social inclusion $38,52,56$

(ii) Meaningful contribution $48,50,52,56$

(iii) Opportunity to reminisce $20,25,29,35-37,41,45,48,52$

(b) Focus on staff

(i) Seeing beyond the illness:

learning personal histories $26,32,40,41$

seeing the person in a family context $28,31,32,34$

dismantling 'us' and 'them'19,23,27,31,32,56

(ii) Examining approach to care:

awareness of approach to care $31,40,41$

opportunities for reflection $26,33,40,53,55$

Conditions required for successful implementation

(a) Reliance on staff

(i) Providing access $20,38,39,47$

(ii) Knowing the person:

provide appropriate encouragement $\mathrm{t}^{30,34,38,47,51}$ provide appropriate reassurance $20,24,25,33,45,48$ tailor the intervention to preferences $29,38,42,49$ tailor the intervention to abilities $23,25,37,40,48,54$

(b) Active involvement of the family $20,28,34,40,49,55$

(c) Flexibility ${ }^{19,24,38,54}$

\section{Challenges to successful implementation}

(a) Pressures on time and staffing $20,24,32,33,39,42,46,47,49,53,55$

(b) Institutional philosophy:

focus on 'priority needs'19,28,32,51

avoidance of risk $27,32,47,51$

(c) Attitudes of staff:

uncomfortable with intervention 24,39,42,47

general resistance $32,53,55$

\section{References}

1 Knapp M, Prince M, Albanese E, Banerjess S, Dhanasiri S, Fernandez J, et al. Dementia UK. Alzheimer's Society, 2007.

2 Kverno KS, Black BS, Nolan MT, Rabins PV. Research on treating neuropsychiatric symptoms of advanced dementia with non-pharmacological strategies, 1998-2008: a systematic literature review. Int Psychogeriatr 2009; 21: $825-43$.

3 APA Work Group on Alzheimer's Disease and other Dementias. Practice guidelines for the treatment of patients with Alzheimer's disease and other dementias. Am J Psychiatry 2007; 164 (suppl 1): 5-56.

4 Fossey J, Ballard C, Juszczak E, James I, Alder N, Jacoby R, et al. Effect of enhanced psychosocial care on antipsychotic use in nursing home residents with severe dementia: a cluster randomised trial. BMJ 2006; 332: 756-8.

5 Vernooij-Dassen $\mathrm{M}$, Vasse $\mathrm{E}$, Zuidema $\mathrm{S}$, Cohen-Mansfield J, Moyle W. Psychosocial interventions for dementia patients in long-term care. Int Psychogeriatr 2010; 22: 1121-8. 
6 O'Connor DW, Ames D, Gardner B, King M. Psychosocial treatments of behaviour symptoms in dementia: a systematic review of reports meeting quality standards. Int Psychogeriatr 2009; 21: 225-40.

7 Craig P, Dieppe P, Macintyre S, Mitchie S, Nazareth I, Petticrew M. Developing and evaluating complex interventions: the new Medical Research Council guidance. BMJ 2008; 337: 979-83.

8 Sandelowski M, Docherty S, Emden C. Qualitative metasynthesis: issues and techniques. Res Nurs Health 1997; 20: 365-71.

9 Campbell R, Pound P, Pope C, Britton N, Pill R, Morgan M, et al. Evaluating meta-ethnography: a synthesis of qualitative research on lay experiences of diabetes and diabetes care. Soc Sci Med 2003; 56: 671-84.

10 Sandleowski M, Barroso J. Toward a metasynthesis of qualitative findings on motherhood in HIV-Positive women. Res Nurs Health 2003; 26: 153-70.

11 Noblit G, Hare RD. Meta-Ethnography: Synthesizing Qualitative Studies. Sage Publications, 1988.

12 Moher D, Liberati A, Tetzlaff J, Altman DG. Preferred reporting items for systematic reviews and meta-analyses: the PRISMA statement. BMJ 2009; 339: 332-6.

13 Mays N, Pope C, Popay J. Systematically reviewing qualitative and quantitative evidence to inform management and policy-making in the health field. J Health Serv Res Policy 2005; 10 (suppl 1): 6-20.

14 Shaw RL, Booth A, Sutton AJ, Miller T, Smith JA, Young B, et al. Finding qualitative research: an evaluation of search strategies. BMC Med Res Methodol 2004; 4: 1-5.

15 Critical Appraisal Skills Programme (CASP). Collaboration for Qualitative Methodologies, 1998 (http://www.casp-uk.net).

16 Dixon-Woods M, Bonas S, Booth A, Jones DR, Miller T, sutton AJ, et al. How can systematic reviews incorporate qualitative research? A critical perspective. Qual Res 2006; 6: 27-44.

17 Britton N, Campbell R, Pope C, Donovan J, Morgan M, Pill R. Using meta ethnography to synthesise qualitative research: a worked example. $J$ Health Serv Res Policy 2002; 7: 209-15.

18 Spalding $M$, Khalsa $P$. Aging matters: humanistic and transpersonal approaches to psychotherapy with elders with dementia. J Humanist Psychol 2010; 50: 142-74.

19 Skovdahl K, Kihlgren AL, Kihlgren M. Dementia and aggressiveness: video recorded morning care from different care units. J Clin Nurs 2003; 12: 888-98.

20 Sixsmith A, Gibson G. Music and the wellbeing of people with dementia. Ageing Soc 2007; 27: 127-45.

21 Perry J, Galloway S, Bottorff JL, Nixon S, Perry J, Galloway S, et al. Nursepatient communication in dementia: improving the odds. J Gerontol Nurs 2005; 31: 43-52.

22 Patton D. Reality orientation: its use and effectiveness within older person mental health care. J Clin Nurs 2006; 15: 1440-9.

23 Palo-Bengtsson L, Ekman S. Emotional response to social dancing and walks in persons with dementia. Am J Alzheimers Dis Other Demen 2002; 17: 149-53.

24 Palo-Bengtsson L, Ekman S. Dance events as a caregiver intervention for persons with dementia. Nursing Inq 2000; 7: 156-65.

25 Palo-Bengtsson L, Winblad B, Ekman S. Social dancing: a way to support intellectual, emotional and motor functions in persons with dementia. J Psychiatr Ment Health Nurs 1998; 5: 545-54.

26 Mckeown J, Clarke A, Ingleton C, Ryan T, Repper J. The use of life story work with people with dementia to enhance person-centred care. Int J Older People Nurs 2010; 5: 148-58.

27 McAllister $\mathrm{CL}$, Silverman MA. Community formation and community roles among persons with Alzheimer's disease: a comparative study of experiences in a residential Alzheimer's facility and a traditional nursing home. Qual Health Res 1999; 9: 65-85.

28 Long-Foley K, Sudha S, Sloane PD, Gold DT. Staff perceptions of successful management of severe behavioural problems in dementia special care units. Dementia 2003; 2: 105-24.

29 Kydd P. Using music therapy to help a client with Alzheimer's disease adapt to long-term care. Am J Alzheimers Dis Other Demen 2001; 16: 103-8.

30 Kovach $\mathrm{CR}$, Henschel H. Planning activities for patients with dementia: a descriptive study of therapeutic activities on special care units. J Gerontol Nurs 1996; 22: 33-8.

31 Kontos $\mathrm{P}$, Mitchell GJ, Mistry B, Ballon B. Using drama to improve person-centred dementia care. J Older People Nurs 2010; 5: 159-68.

32 Kolanowski A, Fick D, Frazer C, Penrod J. It's about time: use of nonpharmacological interventions in the nursing home. J Nurs Scholarsh 2010; 42: 214-22.
33 Kemeny B, Boettcher IF, Deshon RP, Stevens AB, Kemeny B, Boettcher IF, et al. Postintervention focus groups: toward sustaining care. J Gerontol Nurs 2004; 30: 4-9.

34 Kellett U, Moyle W, McAllister M, King C, Gallagher F. Life stories and biography: a means of connecting family and staff to people with dementia. J Clin Nurs 2010; 19: 1707-15.

35 Kawamura N, Niiyama M, Niiyama $\mathrm{H}$. Animal-assisted activity: experiences of institutionalized Japanese older adults. J Psychosoc Nurs Ment Health Serv 2009; 47: 41-7.

36 Katsinas RP. The use and implications of a canine companion in a therapeutic day program for nursing home residents with dementia. Activit Adapt Aging 2000; 25: 13-30.

37 Johnson JR. Effectiveness of memory notebooks upon problematic behavior of residents with Alzheimer's disease. Phys Occup Ther Geriatr 1997; 15: $15-32$.

38 Holthe T, Thorsen K, Josephsson S. Occupational patterns of people with dementia in residential care: an ethnographic study. Scand J Occup Ther 2007; 14: 96-107.

39 Hernandez RO. Effects of therapeutic gardens in special care units for people with dementia: two case studies. J Housing Elderly 2007; 21: 117-52.

40 Hansebo G, Kihlgren M. Patient life stories and current situation as told by carers in nursing home wards. Clin Nurs Res 2000; 9: 260-79.

41 Hagens C, Beaman A, Ryan EB. Reminiscing, poetry writing, and remembering boxes: personhood-centered communication with cognitively impaired older adults. Activit Adapt Aging 2003; 27: 97-112.

42 Gotell E, Brown S, Ekman SL. The influence of caregiver singing and background music on vocally expressed emotions and moods in dementia care: a qualitative analysis. Int J Nurs Stud 2009; 46: 422-30.

43 Gotell E, Brown S, Ekman SL. Influence of caregiver singing and background music on posture, movement, and sensory awareness in dementia care. Int Psychogeriatr 2003; 15: 411-30.

44 Gotell E, Brown S, Ekman SL. Caregiver singing and background music in dementia care. West J Nurs Res 2002; 24: 195.

45 Gotell E, Brown S, Ekman SL. Caregiver-assisted music events in psychogeriatric care. J Psychiatr Ment Health Nurs 2000; 7: 119-25.

46 Gnaedinger N. Changes in long-term care for elderly people with dementia: a report from the front lines in British Columbia, Canada. J Soc Work Long Term Care 2003; 2: 355-71.

47 Gibson G, Chalfont GE, Clarke PD, Torrington JM, Sixsmith AJ. Housing and connection to nature for people with dementia. J Hous Elderly 2007; 21 $55-72$.

48 Gibb H, Morris CT, Glesiberg J. A therapeutic programme for people with dementia. Int J Nurs Pract 1997; 3: 191-9.

49 Gerdner LA. Use of individualized music by trained staff and family: translating research into practice. J Gerontol Nurs 2005; 31: 22.

50 George DR. Intergenerational volunteering and quality of life: mixed methods evaluation of a randomized control trial involving persons with mild to moderate dementia. Qual Life Res 2011; 20: 987-95.

51 Galik EM, Resnick B, Pretzer-Aboff I. 'Knowing what makes them tick': motivating cognitively impaired older adults to participate in restorative care. Int J Nurs Pract 2009; 14: 48-55.

52 Fraser F, James I. Why does doll therapy improve the well-being of some older adults with dementia. PSIEGE Newsletter 2008; 105: 55-63.

53 Emilsson UM. Supervision as pedagogy and support in the Swedish eldercare - a developmental project. J Gerontol Soc Work 2006; 47: 83-102.

54 Eggers T, Norberg A, Ekman S. Counteracting fragmentation in the care of people with moderate and severe dementia. Clin Nurs Res 2005; 14: 343-69.

55 Aveyard B, Davies S. Moving forward together: evaluation of an action group involving staff and relatives within a nursing home for older people with dementia. Int J Older People Nurs 2006; 1: 95-104.

56 Dupuis M, Dobbelsteyn J, Ericson P. Special care units for residents with Alzheimer's (investigating the perceptions of families and staff). Can Nurs Home 1996; 7: 4.

57 Barbour RS. Checklists for improving rigour in qualitative research: a case of the tail wagging the dog? BMJ 2001; 322: 1115-7.

58 Kitwood T. Dementia Reconsidered: The Person Comes First. Open University Press, 1997.

59 Kuske B, Hanns S, Luck T, Matthias C, Angermeyer MC, Behrens J, et al Nursing home staff training in dementia care: a systematic review of evaluated programs. Int Psychogeriatr 2007; 19: 818-41. 
60 Lawrence V, Banerjee S. Improving care in care homes: a qualitative evaluation of the Croydon care home support team. Aging Ment Health 2010; 14: 416-24.

61 Cohen-Mansfield J. Nonpharmacological interventions for inappropriate behaviours in dementia. A review, summary, and critique. Am J Geriatr Psychiatry 2002; 9: 361-81.

62 Cohen-Mansfield J, Libin A, Marx MS. Nonpharmacological treatment of agitation: a controlled trial of systematic individualized intervention. J Gerontol A Biol Sci Med Sci 2007; 62: 908-16.
63 Aylward S, Stolee $\mathrm{P}$, Keat N, Johncox V. Effectiveness of continuing education in long-term care: a literature review. Gerontologist 2003; 43: 259-71.

64 Manthorpe J, Moriarty J. 'Nothing Ventured, Nothing Gained': Risk Guidance for People with Dementia. Department of Health, 2010.

65 Department of Health. Living Well with Dementia: A National Dementia Strategy. Department of Health, 2009

66 Banerjee S. The Use of Antipsychotic Medication for People with Dementia: Time for Action. Department of Health, 2009.

\section{Edward Picot}

Take off that hair;

take off that self-assured air:

take out those teeth;

take that spring from your step and replace it

with a hesitant shuffle.

Take the fresh whites of your eyes

and smudge them yellow,

Take off that sexual appeal:

you won't be needing that any more.

The respect of others: your self-respect too:

they'll have to go.

Put on this extra weight,

these jowls, these liver-spots,

this tremor, these restless nights,

this peevish fretful manner,

this uncertainty, this fear.

this fear

Come on.

You don't think you're ready yet,

but it's time. Your friends are all going.

You're more tired than you know.

And it's too late for another story.

You've been telling yourself a story all day,

acting it out too,

a big exciting fantasy, a real humdinger.

Now the story's over.

come and lie down.

It's getting dark.

This poem is from The Hippocrates Prize 2010: The Winning and Commended Poems, published by The Edge Press. It won the 3rd prize. (c) Edward Picot, personal website http://edwardpicot.com

Chosen by Femi Oyebode. 